Ann. Biol. anim. Bioch. Biophys., I970, 10 (I), I23-I4I.

\title{
VARIATIONS D'EXTRA-CHALEUR INDUITES PAR L'INGESTION DE REPAS TRÈS RICHES EN PROTÉINES CHEZ LE PORC EN CROISSANCE
}

\author{
Geneviève CHARLET-LERY \\ avec la collaboration technique de Marie-Thérèse MoreL \\ Laboratoire de Recherches sur la Conservation et l'Efficacité des Aliments, \\ Centre national de Recherches zootechniques, 78 -Jouy-en-Josas \\ Institut national de la Recherche agronomique
}

\section{RÉSUMÉ}

Le porc en croissance $(30-60 \mathrm{~kg})$ manifeste classiquement après son repas des dépenses énergétiques que nous avons mesurées par calorimétrie indirecte au cours d'une série de confinements de courte durée. L'ingestion brutale d'un repas beaucoup plus riche en protéines que le repas habituel provoque un abaissement de ces dépenses dont la durée est d'environ 7 h et qui s'accompagne d'une chute des coefficients respiratoires.

L'économie d'énergie ainsi réalisée est proportionnelle à la quantité d'azote ingérée en supplément, ou retenue dans l'organisme au cours de la journée de mesure ; elle ne semble pas en corrélation avec l'azote urinaire total, ni avec les fractions uréiques et ammoniacales émises dans la journée. Mais elle pourrait coïncider avec l'apparition dans l'urine d'un métabolite présent dans la fraction non uréique et non ammoniacale. L'augmentation de ce dernier et la diminution des pertes énergétiques seraient simultanées.

\section{INTRODUCTION}

\section{Extra-chaleur et action dynamique spécifique}

L'effet calorigénique des aliments chez l'animal, c'est-à-dire le fait que le sujet qui vient de manger a des dépenses d'énergie plus élevées que s'il est à jeun, est une des notions les plus anciennes du métabolisme énergétique. C'est en effet LAvoISIER qui le mit en évidence peu de temps après avoir montré que la vie était une combustion.

L'importance primordiale de ce phénomène tant au point de vue théorique que pratique, a conduit de très nombreux chercheurs, surtout depuis RUBNER à s'en 
préoccuper. Il n'est pas utile de résumer ici les mises au point très larges et très complètes faites par SCHAEFFER et LEBRETON (I938), par BRODY (I945) et par MIICHELI (I954 et I964). Récemment, KLEIBER (I96I), dans une synthèse très raccourcie, a montré la confusion qui règne parmi les nombreuses hypothèses avancées pour expliquer le mécanisme de ce phénomène.

Tous les auteurs estiment cependant que cet effet représente la partie importante de la différence entre énergie métabolisable et énergie nette et est donc en grande partie responsable de l'abaissement du rendement énergétique de l'organisme animal.

La confusion des idées provient en partie de l'utilisation simultanée de deux vocables souvent employés indifféremment l'un pour l'autre : action dynamique spécifique et extra-chaleur. Pour beaucoup d'auteurs, il semblerait préférable de réserver l'expression action dynamique spécifique ou A.D.S. traduction du Spezifisc dynamische Wirkung de RUBNER aux dépenses d'énergie consécutives à l'ingestion de nutriments simples. (Nous ne discutcrons pas ici, à la suite de l'école anglaise, de l'utilisation erronée du mot " action " qu'il serait préférable de remplacer par le mot " effet ».) Par contre, l'expression " extra-chaleur " devrait être réservée à l'effet calorigénique des rations complexes. Leur mesure est effectuée par différence entre les pertes d'énergie consécutives à l'ingestion d'une ration ou d'un nutriment et celles mesurées pendant la même période chez le sujet n'ayant pas mangé. L'utilisation des deux termes A.D.S. et extra-chaleur, semble indispensable depuis que Forbes et et SWIFT (I944) ont montré que l'extra-chaleur d'une ration comportant plusieurs nutriments est inférieure à la somme des A.D.S. de chacun d'eux. Par contre, effet calorigénique et dépenses post prandiales, utilisées par TERROINE en raison de la période à laquelle se manifeste ce phénomène, sont employés dans un sens moins précis.

Si l'on se réfère à la théorie de Schofnheiner (1942) sur l'état dynamique des constituants corporels, les réactions cataboliques (exothermiques par nature) fournissent de l'énergie libre pour le travail physiologique anabolique : synthèse de glycogène et des graisses et formation des liaisons peptidiques. Mais si cette énergie n'est que partiellement utilisée, la chaleur restante doit être dissipée. Ces déperditions de chaleur consécutives à l'état de réplétion nutritionnelle d'un animal — ou effet calorigénique - s'expliqueraient aisément par cette perte d'énergie libre dans le métabolisme (Mitchei, I, I955).

Toute modification des rapports entre les réactions anaboliques et cataboliques, se répercute sur l'importance de ces pertes. Les causes susceptibles d'induire de telles modifications sont relatives à l'animal ou aux ingesta. Parmi les premières, on peut citer l'état physiologique de l'animal (entretien, lactation, croissance), l'état de ses réserves corporelles (conséquence du régime alimentaire auquel il est soumis - niveau nutritionnel et équilibre de ses constituants - et du moment où est faite la mesture (jeûne plus ou moins long depuis le dernier repas). Les ingesta peuvent varier quantitativement et qualitativement. C'est à ce dernier point qu'apparaît la différence entre A.D.S. et extra-chaleur. Ces multiples causes de variation ont amené BRODY (I945) à conclure qu'il n'y avait pas "une " action dynamique spécifique sauf dans les états physiologiques et nutritionnels étroitement définis. (Cet auteur utilise 1'expression " action dynamique spécifique " dans un sens très large qui inclut la notion d'extra-chaleur).

Il n'est pas possible d'entreprendre une étude sur ce sujet sans prendre en consi- 
dération les causes de variations citées plus haut, afin de préciser rigoureusement les conditions de mesure. En particulier, pour mesurer l'A.D.S. des protides, il nous apparait indispensable de tenir compte de l'influence de la quantité d'énergie ingérée et $\mathrm{du}$ rôle énergétique propre des protides. La référence au jeûne n'éliminant pas ces deux facteurs, nous avons imaginé, à la différence des précédents chercheurs, de comparer à l'extra-chaleur de la ration mixte pauvre en protides (régime à bas niveau d'azote $\mathrm{BN}$ ) que l'animal consomme habituellement à l'extra-chaleur d'un repas équiénergétique mais très riche en azote (repas à haut niveau d'azote $H N$ ), par rapport à la même journée de jeûne. Seul l'apport supplémentaire d'azote - différence entre repas $\mathrm{HN}$ et $\mathrm{BN}$ - peut être la cause des différences enregistrées entre les deux mesures d'extra-chaleur. Une telle mesure représente, à notre avis, 1'A.D.S. de ce supplément d'azote.

La connaissance de l'A.D.S qui précise les relations entre métabolisme azotè et métabolisme énergétique, esst indispensable pour une meilleure compréhension du rôle des protéines animales dans la nutrition.

Jusqu'ici, la grande majorité des travaux sur 1'A.D.S. a été effectuée sur des animaux à l'entretien. Peu ont été faits sur deja animaux en croissance (c'est-à-dire consommant des rations énergétiquement importantes et pour lesquels le supplément d'azote apporté par la ration $H N$ est très important). Nous avons donc appliqué notre méthode au porc en croissance, animal avec lequel nous avons l'habitude d'expérimenter.

\section{MATÉRIEL E'T MÉTHODES}

Un travail antérieur chez le Chien adulte (I96I) nous a montré qu'il n'est pas indispensable de mesurer les extra-chaleurs en elles-mêmes puisque le but de l'expérience est de mesurer leur différence : la seule mesure des dépenses postprandiales consécutives à l'ingestion du repas BN et à celle du repas $H N$ est suffisante. Cette méthode, qui évite de faire régulièrement référence au jeûne, allège le protocole expérimental. C'est ce principe de mesure que nous avons appliqué au porc en croissance.

\section{Les animaux}

Les sujets d'expérience sont des porcs mâles castrés, de race Large White en croissance, dont les poids moyens oscillent de 25 à $60 \mathrm{~kg}$.

Avant le début de tout essai, les animaux sont adaptés à leur nouvel environnement puis aux régimes alternés pendant deux à trois semaines. L'animal vit toujours dans la même cage à bilan, à une température égale à celle de sa neutralité thermique (soit $16-20^{\circ} \mathrm{C}$ pour un sujet en état de réplétion).

\section{Les régimes}

Pour éviter tout phénomène de supplémentation azotée entre régine $\mathrm{BN}$ repas $\mathrm{HN}$, leur équilibre en acides aminés est rigoureusement identique : régime $\mathrm{BN}$ et repas $\mathrm{HN}$ sont constitués par des proportions variables de deux mélanges, l'un protéiprive, l'autre très riche en azote. Ce principe a permis de modifier aussi la richesse en azote des divers régimes BN selon les essais.

La composition centésimale du mélange protéiprive est la suivante : amigel : 50 ; cérélose : 27 ; saccharose : 4 ; papier-filtre : 8 ; matières minérales et vitamines : 8 ; huile : 3 . Celle đu mélange riche en azote : tourteau de soja: 80 ; farine de hareng : 20 . Il est donc légèrement carencé en méthionine.

La teneur en azote des régimes BA, exprimée en pourcentage de la matière sèche (tabl. I, col. 5), a varié de 0,75 (soit environ 4 p. Ioo matières azotées digestibles M.A.D.) - apport qui ne couvre pas les besoins d'entretien des animaux — jusqu'à 3,8 (soit environ 20 p. Ioo M.A.D.) apport qui dépasse largement leurs besoins d'entretien et de croissance. L'azote du repas HN vient donc, soit combler une carence, soit s'ajouter à un excès. 
A titre d'exemple, les régimes à $0,75 \mathrm{p}$. Ioo $\mathrm{N}$ comportent $92 \mathrm{p}$. Ioo de mélange protéiprive et 8 p. roo de mélange azoté, les repas HN à 8,8 p. Ioo N comportent ro p. Ioo de mélange protéiprive et $90 \mathrm{p}$. Ioo de mélange azoté. Dans ces limites et compte tenu de la composition des matières premières, ils apportent des quantités très comparables de matière grasse (de 3 à 3,20 p. Ioo) et de cellulose Weende (de 4,9 à 5,3 p. roo) et sont considérés comme apportant le même nombre de calories digestibles.

TABLEAU I

Résultats moyens selon le repas ingéré par l'animal

\begin{tabular}{|c|c|c|c|c|c|c|c|c|c|}
\hline $\begin{array}{l}\text { No l'orc } \\
\text { (1) }\end{array}$ & Poids & MS & epas & $N$ & $\mathrm{~N}_{i}$ & $\begin{array}{r}\mathrm{N}_{i} \text { en } \\
\text { repa }\end{array}$ & $\begin{array}{l}+ \text { dans } \\
\text { azote }\end{array}$ & $\begin{array}{l}\text { Différ } \\
\text { dépens } \\
\text { diales }\end{array}$ & $\begin{array}{l}\text { ences des } \\
\text { os post pran- } \\
30-420 \mathrm{mn})\end{array}$ \\
\hline 1 & $\begin{array}{c}\mathrm{kg} \\
2\end{array}$ & $\begin{array}{l}g \\
3\end{array}$ & $\begin{array}{c}\mathrm{g} / \mathrm{P}^{0,30} \\
4\end{array}$ & $\begin{array}{c}\% \text { MS } \\
5\end{array}$ & $\begin{array}{c}\text { g/repas } \\
6\end{array}$ & $\begin{array}{c}\text { absolu } \\
\mathrm{g} \\
7\end{array}$ & $\begin{array}{c}g / P^{0,70} \\
g \\
x\end{array}$ & $\begin{array}{c}\text { absolu } \\
\text { kcal } \\
9\end{array}$ & $\mid \begin{array}{c}\% \mathrm{~N}_{i} \text { en }+ \\
\text { kcal } \\
10\end{array}$ \\
\hline $1_{A}(z)$ & 36,2 & $\begin{array}{l}681 \\
692\end{array}$ & 55,6 & $\begin{array}{l}2,67 \\
8,80\end{array}$ & $\begin{array}{l}18,2 \\
60,9\end{array}$ & 12,7 & $3,,^{\prime} 6$ & $+\quad 46,9$ & $+1,10$ \\
\hline $1_{B}\left(f^{\prime}\right)$ & $5: 0$ & $\begin{array}{l}545 \\
550\end{array}$ & 34,5 & $\begin{array}{l}0,73 \\
8,80\end{array}$ & $\begin{array}{r}4,0 \\
48,4\end{array}$ & 14,1 & 2,79 & $\begin{array}{r}+1 \\
+\quad 9,1\end{array}$ & $+0,21$ \\
\hline $2_{A}(5)$ & $10,{ }^{\prime}$ & $\begin{array}{l}748 \\
722\end{array}$ & 54,7 & $\begin{array}{l}3,80 \\
8,20\end{array}$ & $\begin{array}{l}28,4 \\
59,2\end{array}$ & 30,8 & 2,31 & 一 74,0 & $-2,40$ \\
\hline $2_{B}(5)$ & $5: 3,6$ & $\begin{array}{l}744 \\
711\end{array}$ & 41,9 & $\begin{array}{l}0,80 \\
8,10\end{array}$ & $\begin{array}{r}6,0 \\
57,6\end{array}$ & 51,6 & 3,18 & $-123,0$ & $-\quad 2,39$ \\
\hline $3_{A}(5)$ & $3: y^{\prime}$ & $\begin{array}{l}573 \\
576\end{array}$ & 49,5 & $\begin{array}{l}0,80 \\
8,55\end{array}$ & $\begin{array}{r}4,6 \\
49,3\end{array}$ & 14,7 & $3,8^{\prime}+$ & $-92,9$ & $-2,08$ \\
\hline $3_{B}(5)$ & 52,2 & $\begin{array}{l}992 \\
959\end{array}$ & 61,3 & $\begin{array}{l}2,55 \\
8,55\end{array}$ & $\begin{array}{l}25,3 \\
8 \because, 0\end{array}$ & 56,7 & 3,57 & $-150,0$ & $-2,65$ \\
\hline $4_{\mathrm{A}}(3)$ & $3 \geq, 9$ & $\begin{array}{l}623 \\
616\end{array}$ & 53,7 & $\begin{array}{l}0,81 \\
8,61\end{array}$ & $\begin{array}{r}5,0 \\
53,0\end{array}$ & 48,0 & 4,16 & $-129,2$ & $-2,69$ \\
\hline${ }_{1} \mathrm{~B}(\overline{3})$ & 61,7 & $\begin{array}{l}812 \\
838\end{array}$ & 57,8 & $\begin{array}{l}2,67 \\
8,18\end{array}$ & $\begin{array}{l}21,7 \\
71,1\end{array}$ & 49,1 & 3,46 & $-118,9$ & — 2,11 \\
\hline $\begin{array}{r}5_{\text {A }}(i) \\
\text { B ( }(a)\end{array}$ & $60, \dot{x}$ & $\begin{array}{ll}1 & 116 \\
1 & 130 \\
1 & 105\end{array}$ & 63,0 & $\begin{array}{l}0,77 \\
4,13 \\
8,47\end{array}$ & $\begin{array}{r}8,6 \\
46,7 \\
93,6\end{array}$ & $\begin{array}{l}38,1 \\
85,0\end{array}$ & $\begin{array}{l}2,16 \\
4,8=1\end{array}$ & $\begin{array}{r}7: 0 \\
-\quad 218,3\end{array}$ & $\begin{array}{l}-1,9 \mathbf{t} \\
-2,57\end{array}$ \\
\hline $6 \quad(5)$ & $46,{ }^{\prime}$ & $\begin{array}{l}856 \\
84: 4\end{array}$ & 58,1 & $\begin{array}{l}2,16 \\
7,73\end{array}$ & $\begin{array}{l}18,5 \\
65,2\end{array}$ & 46,7 & 3,19 & $-8: 3,5$ & $-1,79$ \\
\hline 7 (5) & 37,6 & $\begin{array}{l}421 \\
4 \geq 1\end{array}$ & 41,8 & $\begin{array}{l}1,21 \\
7,80\end{array}$ & $\begin{array}{r}5,1 \\
32,8\end{array}$ & 27,9 & 2,78 & - $68,9 *$ & $-2,47$ \\
\hline
\end{tabular}

(1) entre parenthèses nombre de données.

${ }^{*}{ }^{*}$ valeur approximative extrapolée à partir des consommations d'O 2 .

En italique : valeurs relatives aux repas $B N$.

Les quantités d'ingesta $\mathrm{BN}$ ont été limitées par l'appétit des animaux pour le repas $\mathrm{HN}$ puisque, par définition, les quantités ingérées doivent être identiques. A fin que les mesures soient rigoureusement comparables, elles sont faites uniquement les jours où l'animal ingère la totalité de sa ration. Il n'est pas distribué plus de deux repas HN par semaine pour éviter toute possibilité d'interaction d'un repas sur l'autre. Le repas du soir est toujours un repas BN. 
Les journées d'expérimentation sont toujours couplées par deux, celle du repas BN précède obligatoirement celle du repas HN. Dans l'essai 5, une seule mesure pour le repas BN a servi de point de comparaison pour un repas HN à 4 , I p. Ioo, normalement couplé et pour un repas à 8 p.roo distribué 2 jours plus tard. Les repas sont distribués à heure fixe : $9 \mathrm{~h} \mathrm{I}_{5}$ et $\mathrm{I} 7 \mathrm{~h} 30$.

Les rations sont ajustées régulièrement en fonction du poids de l'animal. Lorsqu'il y a modifications du taux d'azote du régime $\mathrm{BN}$, la phase d'adaptation dure au minimum huit jours.

\section{Bilans azotés ingesta-excreta}

Les ingesta sont mesurés rigoureusement par pesée des quantités distribuées et refusées.

Les excreta. - Nous n'avons pas prévu de collecter les fèces, les cœfficients de digestibilité du mélange azoté et du mélange protéiprive nous ayant été donnés par des expériences précédentes. Il nous a semblé possible de les utiliser sans commettre d'erreur importante.

Par contre, l'ingestion brutale de grosses quantités d'azote provoque l'émission importante d'azote urinaire qu'il est indispensable de mesurer rigoureusement. Les urines sont collectées soit sur la totalité des $24 \mathrm{~h}$, soit séparément entre $9 \mathrm{~h} \mathrm{I}_{5}$ et $\mathrm{I} 8 \mathrm{~h}$ et entre $\mathrm{I} 8 \mathrm{~h}$ et $9 \mathrm{~h} \mathrm{I} 5$. La collecte commence avec la première miction qui suit le repas.

Cette différenciation des émissions entre jour et nuit nous a apparu insuffisante et il nous a semblé nécessaire d'effectuer des mesures plus fréquentes. Pour contrôler l'évolution au cours de la journée, nous avons collecté toutes les 2 heures l'urine d'un certain nombre d'animaux soumis aux régimes précédemment utilisés, mais sur lesquels nous n'avons pas mesuré les dépenses d'énergie :

- Porc $\mathrm{X}(5 \mathrm{I} \mathrm{kg})$ soumis aux conditions de l'animal 2, essai A (régime BN et repas HN respectivement 3,90 et $8,66 \mathrm{~N}$ p. Ioo m.s.). Les mesures ont été effectuées en triple.

- 3 porcs $(\mathrm{N}=50 \mathrm{~kg}, \mathrm{P}=56 \mathrm{~kg}, \mathrm{R}=56 \mathrm{~kg})$ soumis aux conditions de l'animal 5 , essais A et $\mathrm{B}$ (régime $\mathrm{BN}: 0,8 \mathrm{~N}$ p. Ioo m.s., repas $\mathrm{HN}: 4$ et $8 \mathrm{~N}$ p. Ioo m.s.). Une seule journée a été contrôlée pour chaque type de repas.

Les dosages d'azote sont faits par micro Kjeldahl. Ceux d'urée par la méthode de Schramm et Aines, et ceux d'ammoniaque par celle de Conway. Ces derniers dosages ont été effectués sur les émissions individuelles des 3 porcs, immédiatement après leur collecte.

\section{Mesures d'énergie et mode d'expression}

Les mesures énergétiques sont faites par calorimétrie indirecte en confinement. La cage à confinement, les méthodes de collecte des échantillons, celles d'analyse des gaz $\mathrm{CO}_{2}$ et $\mathrm{O}_{2}$ ainsi que le calcul des consommations $\mathrm{d}^{\prime} \mathrm{O}_{2}$ et de rejet de $\mathrm{CO}_{2}$ ont été précédemment décrits (CHARLETLERY, I958 $a$ et $b$ ). Nous précisons seulement que la durée de chaque confinement est suffisamment courte pour que le seuil de I p. Ioo de $\mathrm{CO}_{2}$ ne soit jamais atteint.

Comme déjà précisé, nous avons uniquement mesuré les dépenses énergétiques postprandiales consécutives à l'ingestion des repas BN et $\mathrm{HN}$.

Consécutivement au repas du matin, 6 et 8 confinements sont effectués : le premier vers 10 heures, le dernier vers $16 \mathrm{~h}$ 30. Ils n'ont lieu que si les sujets sont calmes et couchés afin d'éviter les corrections de position debout toujours aléatoires. Pour pallier les irrégularités inévitables des moments où ils sont effectués, on calcule par intrapolation à partir des valeurs observées les valeurs $\mathrm{d}^{\prime} \mathrm{O}_{2}$ consommé et de $\mathrm{CO}_{2}$ rejeté aux temps 60 o 90 I 20 I 80240300420 minutes après le milieu du repas, les quantités d'énergie dépensée à ces divers temps sont calculées par la formule de Brouwer (1958).

Nous avons essayé de répéter chaque couple de mesure (repas BN- repas HN) cinq fois, mais le comportement des animaux n'a pas toujours permis d'atteindre ce nombre. Le nombre de couples obtenus - entre 2 et 5 - est indiqué au tableau I (col. I).

Les courbes moyennes représentant l'évolution des dépenses d'énergie en fonction du temps sont tracées, pour les deux séries de mesure, sur un même graphique, ce qui permet l'évaluation par planimètre de la différence. Les résultats ainsi obtenus recoupent rigoureusement les données çalculées par le procédé inverse : évaluation par planimètre des différences de consommation $\mathrm{d}^{\prime} \mathrm{O}_{2}$ et de production de $\mathrm{CO}_{2}$ et calcul ultérieur de la différence d'énergie.

\section{RÉSULTATS}

Les résultats de ce travail ont été déjà sommairement exposés lors du IX ${ }^{\mathrm{e}}$ Congrès de Zootechnie (Charlet-Lery, I966). 


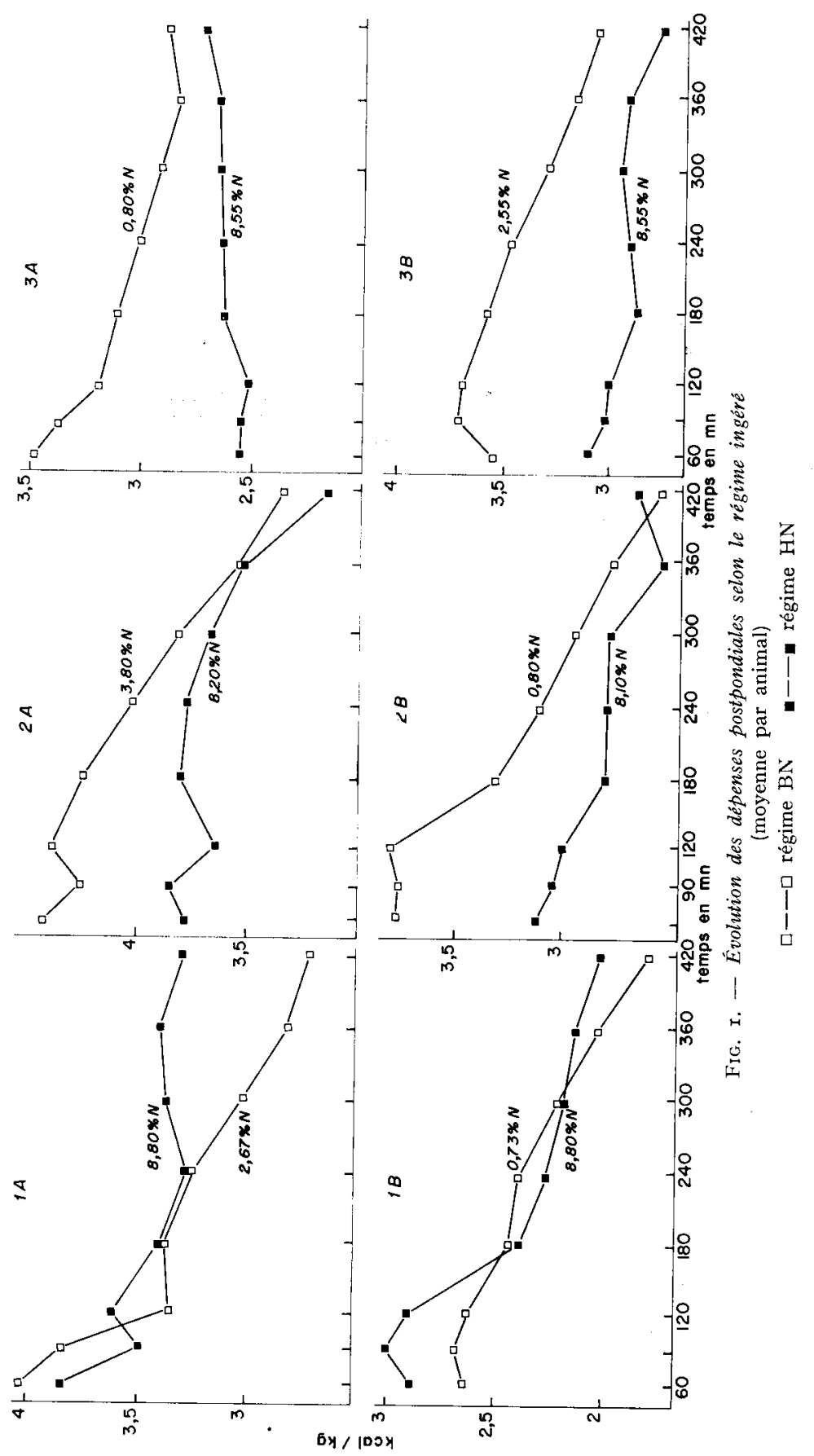




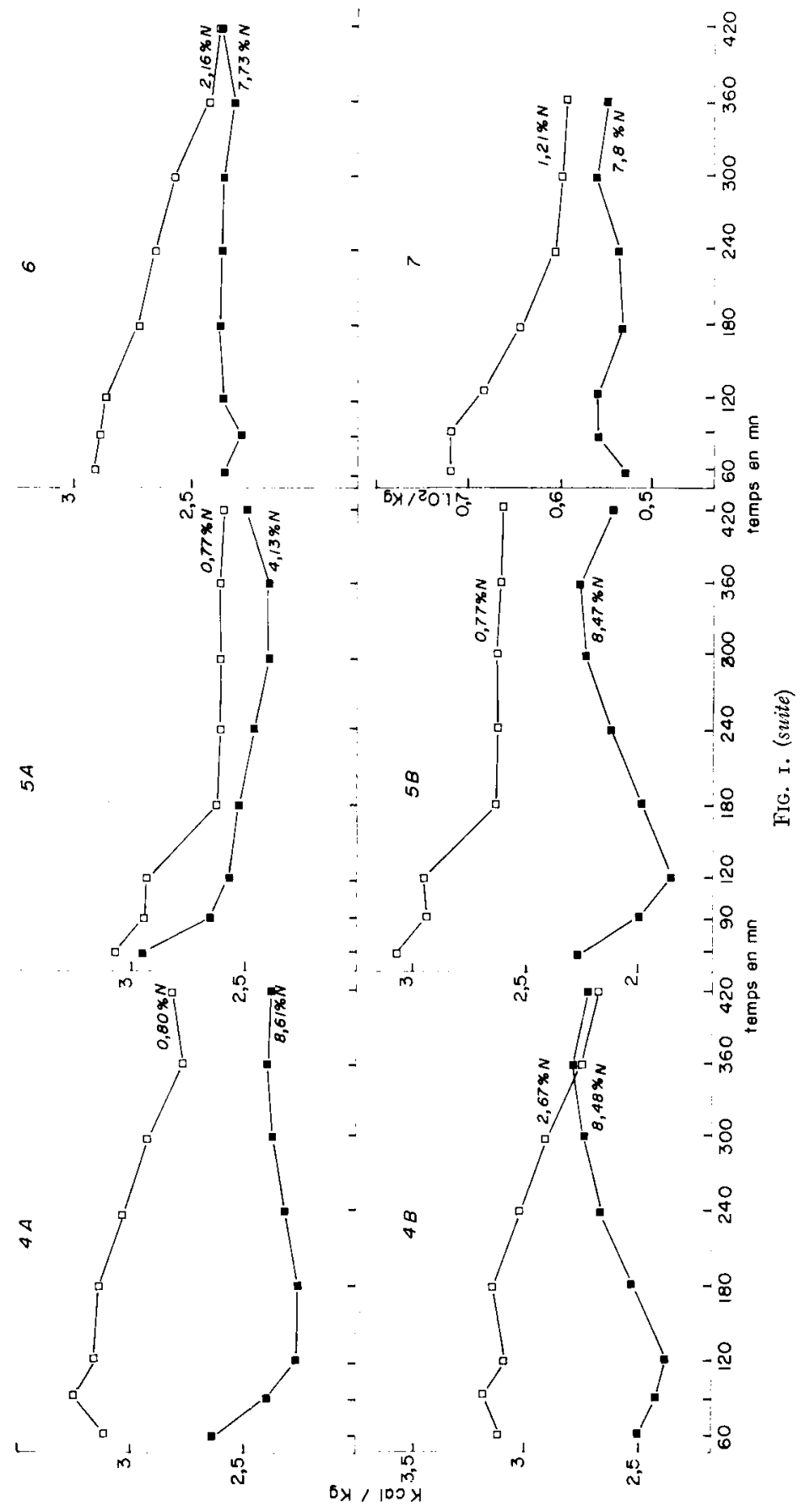


Les animaux s'adaptent rapidement à ce type d'alimentation. Par suite du niveau énergétique suffisamment élevé de leurs rations, ils font une croissance pondérale normale pour des porcs en croissance de ce type. Par suite de l'ingestion environ 2 fois par semaine de repas HN, les gains dépassent $300 \mathrm{~g}$ par jour lorsque le régime $\mathrm{BN}$ n'apporte que $0,7-0,8 \mathrm{p}$. I0o $\mathrm{N}$ et varient entre 470 et $75^{\circ} \mathrm{g}$. dans les autres cas. On peut donc considérer que nos animaux sont en croissance.

Le tableau I indique les quantités moyennes de matière sèche consommées par l'animal au repas du matin selon qu'on lui offre son régime BN ou son repas $\mathrm{HN}$ (col. 3). L'égalité presque totale entre les ingesta permet d'assurer, puisque les rations sont équi-énergétiques, que les animaux ont ingéré les mêmes quantités d'énergie.

Le supplément d'azote apporté par le repas HN $(\mathrm{g} \mathrm{Ni}+)$ est calculé à la colonne 7 .

La figure I montre, pour chaque animal, l'évolution des dépenses d'énergie selon ses ingesta. Les différences d'énergie calculées par planimètrie sont portées à la colonne 9 .

De l'étude de ces données, il ressort trois points :

a) Du point de vue méthodologique, la durée choisie de 420 minutes est suffisamment longue puisque, dans la majorité des cas, les deux courbes ont tendance à se rejoindre ou se rejoignent complètement entre 360 et 420 minutes, ce qui fait supposer que le phénomène observé, c'est-à-dire les différences d'extra-chaleur, est terminé.

b) Contrairement aux résultats que nous avons obtenus sur le Chien (I96I), et aux hypothèses qui avaient été formulées alors, l'ingestion d'un repas HN conduit dans la grande majorité des essais (Io fois sur I2, soit 6 animaux sur 7), non pas à une augmentation mais à une diminution des dépenses d'extra-chaleur (col. 9 et fig. I) et ce, quel que soit le niveau azoté du régime BN, inférieur, égal ou supérieur aux besoins de l'animal. Au cours d'un même essai, les réactions de l'animal sont homogènes, soit toujours positives, soit toujours négatives. Aussi, malgré les variations journalières, la différence entre les courbes d'évolution des dépenses est presque toujours significative au seuil de 5 p. Ioo, souvent à celui de I p. Ioo aux temps de 6090, I20 et I 80 minutes.

Quatre autres animaux ont été ultérieurement soumis au même type de régime et ont réagi d'une façon analogue.

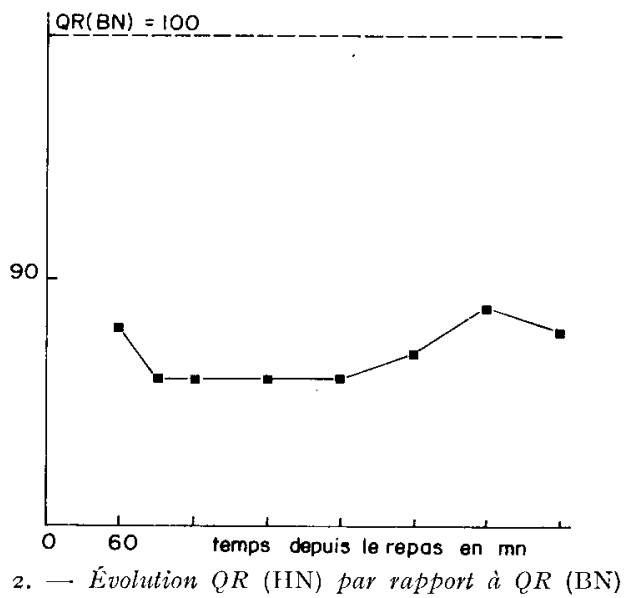


c) Cette diminution des dépenses posprandiales, s'accompagne d'une baisse des quotients respiratoires (tab1. 3) qui est relativement constante au cours de la journée, (et que l'on constate souvent avec plus d'ampleur sur les valeurs brutes). Cette baisse semblerait caractéristique de l'animal et non de son régime comme on le constate chez les animaux qui ont reçu successivement 2 régimes $B N$ différents $\left(2_{\Lambda}\right.$ et $2_{13}$ respectivement -4 et -5 p. Iоо ; 3 A et 3 в : - I6 et -20 p. I00; 4 A et 4 n : - I6 et - I I p. roo). Elle reste en moyenne, relativement constante entre 60 et 420 minutes (fig. 2) et ne paraît pas s'atténuer.

Ie plus, nous avons remarqué que dans les quelques heures qui suivent le repas $\mathrm{HN}$, les porcs sont particulièrement calmes et semblent plongés dans un sommeil profond qui facilite beaucoup les mesures d'énergie.

Il semble logique d'isoler le cas du porc I qui a réagi à l'inverse des autres et dont nous indiquons cependant les résultats. Les calculs ont été faits uniquement avec les sujets pour lesquels le repas $\mathrm{HN}$ a provoqué une moindre extra-chaleur que le régime $\mathrm{BN}$.

\section{Relation avec l'azote ingéré}

Chez les 6 porcs, (Io essais), l'économie de calories est en corrélation élevée et hautement significative avec la quantité d'azote ingéré en supplément $(\mathrm{N} i+)$ :

$$
r=+0,98 \pm 0,013
$$

La régression linéaire qui lie ces deux séries de valeurs est :

$$
\begin{aligned}
& y=2,73 x+\mathrm{I} 7 \\
& x=\mathrm{g} . \mathrm{N} i+ \\
& y=\text { kcal économisées }
\end{aligned}
$$

Les mêmes données rapportées à la taille métabolique conduisent à un coefficient de corrélation du même ordre :

$$
r=+0,9 \mathrm{I} \pm 0,04 .
$$

Dans les conditions où ont été placés les animaux $[\mathrm{g} \mathrm{N} i+: 28$ à 85 en valeur absolue soit 2,3 à 4,8 par rapport à la taille métabolique $(\mathrm{P} 0,7 \mathrm{O})$ ], on peut considérer que l'économie de calories par g Ni + est constante (col. Io), elle s'élève, à $2,34 \mathrm{kcal}$ \pm o, Io (erreur standard).

\section{Relation avec l'excrétion urinaire d'azote}

La consommation d'un repas HN provoque une augmentation importante de l'excrétion urinaire d'azote, dans les heures qui suivent le repas (tabl. 2, col. 4 et 8). Mais il n'existe que des corrélations très faibles entre économie d'énergie et excrétion supplémentaire d'azote urinaire ( 9 -I $8 \mathrm{~h}$ ), que celles-ci soient considérées en valeurs absolues ou rapportées à la taille métabolique. I)'ailleurs cette augmentation se prolonge très au-delà du phénomène considéré (col. 6 et Io) : les suppléments horaires d'azote urinaire étant dans la majorité des cas, égaux ou plus élevés au cours de la nuit suivante (tab1. 2, col. 9 et I I).

L'importance de l'émission horaire d'azote au cours de la nuit qui suit la journée HN ressort aussi des données obtenues avec les porcs $X$ et N.P.R. qui ont respecti- 
TABLEAU 2

Emission d'azote urinaive

(moyenne par animal)

\begin{tabular}{|c|c|c|c|c|c|c|c|c|c|c|c|c|}
\hline \multirow{3}{*}{$\begin{array}{c}\text { No } \\
\text { Porc }\end{array}$} & \multirow{2}{*}{\multicolumn{2}{|c|}{$\begin{array}{l}\text { N digéré en }+ \\
\text { dans } \\
\text { repas azoté }\end{array}$}} & \multicolumn{4}{|c|}{$\mathrm{N}$ urinaire } & \multicolumn{4}{|c|}{$\begin{array}{l}N \text { urine émis en }+ \\
\text { après repas azoté }\end{array}$} & \multirow{2}{*}{\multicolumn{2}{|c|}{$\begin{array}{c}\begin{array}{c}\mathrm{N} \text { appar. } \\
\text { retenu }\end{array} \\
9-18 \mathrm{~h}\end{array}$}} \\
\hline & & & \multicolumn{2}{|c|}{$9-18 \mathrm{~h}$} & \multicolumn{2}{|c|}{$18-9 h$} & \multicolumn{2}{|c|}{$9-1 \times h$} & \multicolumn{2}{|c|}{$18-9 h$} & & \\
\hline & $g$ & $\mathrm{~g} / \mathrm{P}^{\mathbf{0}, 70}$ & $g$ & $\mathrm{~g} / \mathrm{h}$ & $g$ & $\mathrm{~g} / \mathrm{h}$ & $g$ & $g / h$ & $g$ & $\mathrm{~g} / \mathrm{h}$ & $g$ & $g / P^{0}, 80$ \\
\hline 1 & 2 & 3 & i & 5 & 6 & 7 & 8 & 9 & 10 & 11 & 12 & 13 \\
\hline $1_{\mathrm{A}}$ & $\overline{36 ;, 3}$ & $-\overline{2,95}$ & $\begin{array}{r}5,18 \\
10,33\end{array}$ & $\begin{array}{l}0,58 \\
1,15\end{array}$ & & & 5,15 & & & & & \\
\hline $1_{\mathrm{B}}$ & $\overline{37,7}$ & $-\overline{2,37}$ & $\begin{array}{l}1,1 t \\
6,32\end{array}$ & $\begin{array}{l}0,1.3 \\
0,70\end{array}$ & & & 5,18 & & & & & \\
\hline $2_{A}$ & - & $\overline{1,96}$ & $\begin{array}{l}10,04 \\
17,79\end{array}$ & $\begin{array}{l}1,12 \\
1,98\end{array}$ & $\begin{array}{l}17,50 \\
27,96\end{array}$ & $\begin{array}{l}1,17 \\
1,86\end{array}$ & 7,75 & $0,8 i$ & 10,46 & 0,70 & $18, i$ & 1,38 \\
\hline $2 B$ & $\overline{13,8}$ & $\overline{2,70}$ & $\begin{array}{l}2,06 \\
9,19\end{array}$ & $\begin{array}{l}0,2.3 \\
1,02\end{array}$ & $\begin{array}{r}2,99 \\
16,0 \text { t }\end{array}$ & $\begin{array}{l}0,20 \\
1,07\end{array}$ & 7,13 & 0,79 & 13,05 & 0,87 & 36,7 & $2, \geq 6$ \\
\hline $3_{A}$ & 38,0 & $\overline{3,27}$ & $\begin{array}{l}1,39 \\
5,21\end{array}$ & $\begin{array}{l}0,1 ; \\
0,58\end{array}$ & $\begin{array}{r}1,87 \\
12,96\end{array}$ & $\begin{array}{l}0,12 \\
0,8^{\prime}\end{array}$ & 3,82 & $0, t^{\prime} 3$ & 11,09 & $0,7 / 4$ & 34,2 & 2,96 \\
\hline $3_{\mathrm{B}}$ & $\overline{18,2}$ & $\overline{3,03}$ & $\begin{array}{r}9,5.3 \\
17,92\end{array}$ & $\begin{array}{l}1,06 \\
2,00\end{array}$ & $\begin{array}{l}13,84 \\
33,41\end{array}$ & $\begin{array}{l}0,32 \\
2,333\end{array}$ & 8,39 & 0,93 & 19,57 & 1,30 & 39,8 & 2,50 \\
\hline $1 \mathrm{~A}$ & $\overline{40,8}$ & $\overline{3,53}$ & $\begin{array}{l}1,57 \\
7,17\end{array}$ & $\begin{array}{l}0,17 \\
0,8: 3\end{array}$ & $\begin{array}{r}1,82 \\
12,81\end{array}$ & $\begin{array}{l}0,1: 2 \\
0,85\end{array}$ & 5,90 & 0,66 & 10,99 & 0,73 & $3 / 4,9$ & 3,00 \\
\hline $4 \mathrm{~B}$ & - & $\overline{2, y^{\prime}}$ & $\begin{array}{r}8,54 \\
16,81\end{array}$ & $\begin{array}{l}0,95 \\
1,87\end{array}$ & $\begin{array}{l}13,61 \\
31,61\end{array}$ & $\begin{array}{l}0,91 \\
2,11\end{array}$ & 8,27 & 0,92 & 18,00 & $1, \geq 0$ & 33,7 & 2,36 \\
\hline $5_{\mathrm{A}}$ & $\begin{array}{l}- \\
32,4 \\
72,2\end{array}$ & $\begin{array}{l}-\overline{1,8} \\
1,09\end{array}$ & $\begin{array}{l}1,64 \\
4,82 \\
8,15\end{array}$ & $\begin{array}{l}0,18 \\
0,5^{\prime} \\
0,94\end{array}$ & $\begin{array}{r}2,55 \\
7,26 \\
13,92\end{array}$ & $\begin{array}{l}0,17 \\
0,18 \\
0,013\end{array}$ & $\begin{array}{l}3,18 \\
6,8\end{array}$ & $\begin{array}{l}0,36 \\
0,76\end{array}$ & $\begin{array}{r}4,71 \\
11,37\end{array}$ & $\begin{array}{l}0,36 \\
0,76\end{array}$ & $\begin{array}{l}29,2 \\
65,1\end{array}$ & $\begin{array}{l}1,65 \\
3,80\end{array}$ \\
\hline
\end{tabular}

En italique : valeurs relatives aux repas $B N$.

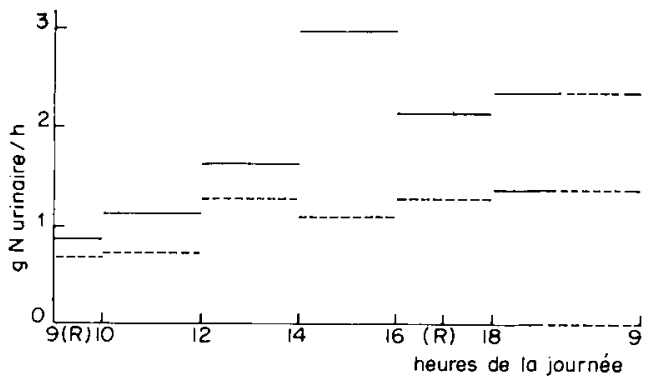

FIc. 3. - Evolution de l'excrétion $N$ urinaire au cours de la journée en fonction du régime (Porc $\mathrm{X}$ ) 
vement consommé les mêmes rations que les porcs $2 \mathrm{~A}$ et $5 \mathrm{~A}$ et $\mathrm{B}$. En effet, pour ces 4 animaux, l'ingestion du repas $H N$ provoque rapidement une excrétion d'azote importante, mais cette excrétion s'intensifie au cours de la journée et reste très élevée toute la nuit, bien que le soir, les animaux ne consomment qu'un repas BN. La figure 3 pour le porc $X$, la figure 4 pour la moyenne des 3 porcs N.P.R. montrent nettement cette évolution.

Si l'on considère la fraction azote uréique et ammoniacal, son excrétion évolue dans le temps et en intensité relative à peu près comme celle de l'azote urinaire totale.

Par contre, si l'on considère l'azote non uréique et non ammoniacal, que l'on calcule par différence, son excrétion, à la suite des repas $\mathrm{HN}$, évolue de façon différente. Importante dans les premières deux heures, elle s'intensifie dans les deux heures suivantes mais elle a ensuite tendance à s'atténuer et après $I 8$ heures et au cours de la nuit revient à des valeurs très proches de celles observées lorsqu'il y a ingestion d'un repas $\mathrm{BN}$ le matin.

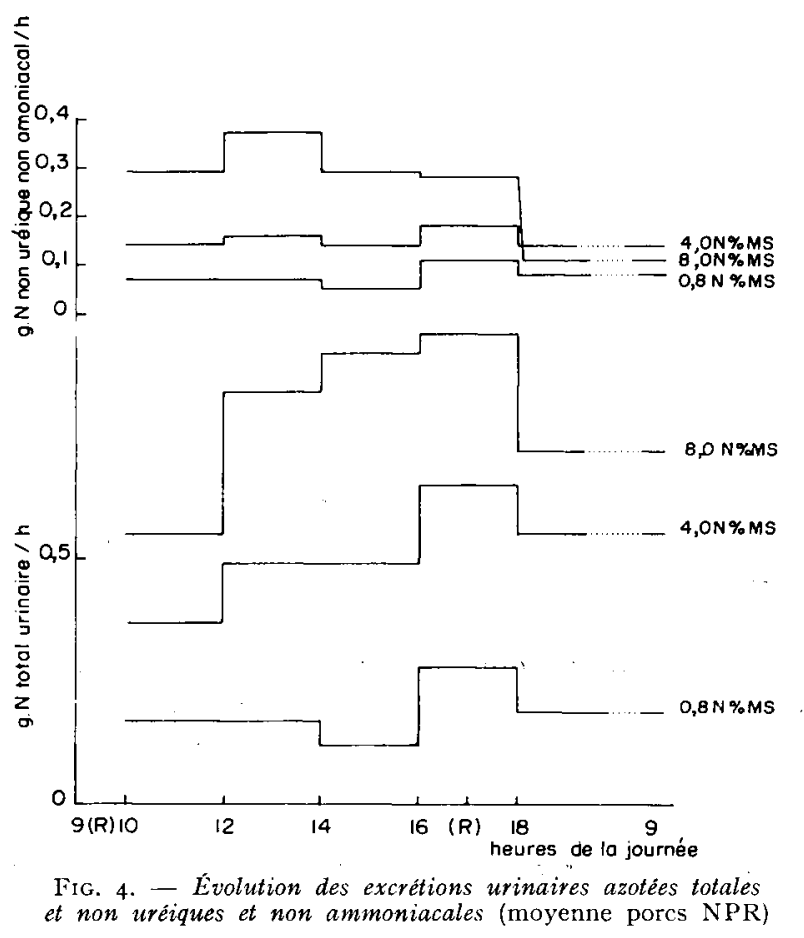

\section{Relation avec l'azote apparemment retenu}

Enfin, nous avons essayé de calculer la quantité d'azote supplémentaire apparemment retenue au niveau de l'organisme pendant la journée. N'ayant pas mesuré les coefficients de digestibilité (CUD) de l'azote dans nos conditions expérimentales, nous avons admis que l'azote ingéré en supplément a un coefficient égal à celui observé pour des rations de même composition mais dont la teneur en azote est constante : 
soit 85 p. Ioo. Cette hypothèse n'est pas contredite par les résultats de Yeo et CHAMBERLAIN (I966) qui n'observent pas de modification du CUD $N$ lorsqu'ils dissocient les apports d'énergie (le matin) et de protéine (le soir) chez les porcs en croissance $(15-55 \mathrm{~kg}) \mathrm{ni}$ par nos propres résultats ultérieurs (I970).

De cet azote supplémentaire théoriquement digéré (tab1. 2, col. 2), on déduit l'azote urinaire supplémentaire (col. 7) pour obtenir l'azote apparemment retenu en plus au niveau de l'organisme entre 9 et $\mathrm{I} 8 \mathrm{~h}$ (col. 9).

Le coefficient de corrélation entre cette dernière quantité et l'économie de calories atteint la valeur suivante:

$$
r=+0,93 \pm 0,05
$$

La droite de régression passe par l'origine et l'économie de calories est dans ce cas proportionnelle à la quantité d'azote retenu en plus, consécutivement au repas HN.

$$
y=3,35 x
$$

$y=\mathrm{kcal}$ économisées entre 60 et 420 minutes.

$x=\mathrm{g} \mathrm{N}$ apparemment retenu en plus dans l'organisme (9 h $\mathbf{I} 5^{-\mathbf{I}} 8 \mathrm{~h}$ ).

Si les deux séries de données sont rapportées à la taille métabolique, le cofficient est encore plus élevé :

$$
r=+0,98 \pm 0,02
$$

\section{DISCUSSION}

Rappelons que pour mesurer l'A.D.S. des protides, il nous a paru indispensable de tenir compte de l'influence de la quantité d'énergie ingérée et du rôle énergétique propre des protides. Nous avons donc comparé à l'extra-chaleur de la ration mixte pauvre en protides (BN) que l'animal ingère habituellement, l'extra-chaleur du repas riche $(\mathrm{HN})$. Les différences d'extra-chaleur constituent 1'A.D.S. du supplément d'azote ingéré $(\mathrm{N} i+)$. Dans ces conditions elles ne peuvent être imputables qu'à ce nutriment.

Cette méthode a été appliquée à des animaux dont les niveaux d'ingestion sont très différents : d'abord adultes à l'entretien, ensuite jeunes en croissance, afin d'obtenir une gamme aussi large que possible des valeurs $\mathrm{N} i+$. Des raisons matérielles et méthodologiques nous ont obligés à recourir à deux espèces différentes : le Chien comme animal à l'entretien, le Porc comme animal en croissance, susceptibles l'un et l'autre d'ingérer des repas $\mathrm{HN}$ et $\mathrm{BN}$ de richesse azotée très variable. Les niveaux d'ingestion par repas et rapportés à $\mathrm{P}$ o,70 varient pour les chiens de Io à $20 \mathrm{~g}$ et pour les pores de 35 à $63 \mathrm{~g}$; les variations de $\mathrm{N} i$ + sont pour les premiers de 0,26 à $\mathrm{I}, 9 \mathrm{I}$ et pour les seconds de $2, \mathrm{I} 6$ à $4,82 \mathrm{~g}$.

Les 3 chiens utilisés nous ont montré (I96r), comme cela était prévisible d'après tous les travaux antérieurs, que l'extra-chaleur des repas HN (viande dégraissée) était supérieure à celle des repas $\mathrm{BN}$ (viande dégraissée et riz). Mais ayant fait varier la richesse en azote des repas $B N$, nous avons constaté que le nombre de calories supplémentaires perdues par $\mathrm{g}$ d'N $i+$, donc l'A.D.S., était d'autant plus élevé que le régime $\mathrm{BN}$ était lui-même plus riche en azote. On pouvait donc, selon la théorie de 
MitcheiL (I955), relier l'augmentation de l'A.D.S. des protides à une perte d'énergie libérée par un catabolisme azoté accru : en effet, il serait normal que le chien adulte à l'entretien dont les besoins azotés sont couverts, soit inapte à fixer un supplément d'azote.

Chez le porc en croissance, entre 30 et $60 \mathrm{~kg}$, le phénomène enregistré est inverse. Les dépenses énergétiques consécutives à l'ingestion des repas $\mathrm{HN}$ plus faibles que celles consécutives aux repas $\mathrm{BN}$ (1) et par $\mathrm{g} \mathrm{N} i+$ on constate une économie de calories. Telle que nous 1'avons définie, il n'y aurait pas d'A.I.S. chez le porc en croissance mais une économie de calories.

L'économie d'énergie est proportionnelle au supplément d'azote ingéré $(2,34$ \pm 0$, Io $\mathrm{kcal} / \mathrm{g} \mathrm{N} i+; r=+0,98)$ et simultanément à la quantité d'azote retenu au niveau de l'organisme pendant la même période $(3,35 \mathrm{kcal} / \mathrm{g} \mathrm{N}$ digéré +$)$.

Ce phénomène observé dans des conditions expérimentales très particulières apparaît contraire aux connaissances que l'on possède sur les métabolismes énergétiques et azotés et ne semble pas avoir été précédemment signalé. Cependant, le nombre de fois oì il a été observé et la signification des résultats malgré leur petit nombre pour chaque animal, montre sa régularité et son importance.

Afin de pouvoir rechercher des hypothèses susceptibles de l'expliquer, nous allons, à partir des résultats précédemment exposés, essayer de le caractériser.

\section{I. - Relation entre économie de calories et métabolisme azoté}

\section{Azote urinaire total - azote et uréique ammoniacal.}

La corrélation entre économie de calories et quantité d'azote urinaire émise en plus par l'animal pendant la même journée (entre 9 et $\mathrm{I} 8 \mathrm{~h}$ ) est faible et non significative. La collecte des émissions urinaires dès la première heure était difficile à éviter du point de vuc pratique : elle n'entraîne qu'une légère erreur. En effet, élaboration de l'urine et miction n'étant pas simultanées, la première miction après le repas présente encore un faible taux azoté correspondant à la phase de jê̂ne nocturne. Mais la quantité d'azote apportée par celle-ci ne représente que $5 \mathrm{p}$. Ioo du total 9-I 8 h (résultats moyens du porc $\mathrm{X}$ ) et ne peut donc pas modifier les résultats. Cette non simultanéité nous a conduit à collecter l'urine après l'arrêt des mesures d'énergie, jusqu'à $I 8 \mathrm{~h}$, soit une ou deux mictions, dont celle qui suit le repas du soir.

Si l'on admet que les porcs N.P.R. soumis aux mêmes régimes que le porc 5 aient eu les mêmes dépenses d'énergie que ce dernier et qu'inversement celui-ci ait présenté les mêmes émissions urinaires que la moyenne des 3 autres, on confirme l'absence de corrélation susdite. En effet, après les repas HN, les excrétions horaires d'azote total s'intensifient en fin de journée tandis que les dépenses énergétiques du porc 5 reviennent au niveau du repas $\mathrm{BN}$ (fig. 4). Il en est de même pour le porc $\mathrm{X}$ qui a reçu le même régime que le porc $3 \mathrm{~B}$.

Les fractions uréiques et ammoniacales ont été dosées dans les diverses émissions des porcs N.P.R. ; elles varient comme l'azote urinaire total dont elles représentent une grande partie. Il semble logique d'arriver aux mêmes conclusions, que l'on considère l'un ou l'autre.

( ${ }^{1}$ Un animal a réagi à l'inverse des autres et ses données ont été éliminées des calculs. 
En outre, l'observation des données recueillies montrent que l'azote apporté par le repas HN n'est que lentement éliminé. A I $8 \mathrm{~h}$ les animaux n'ont éliminé que 20 p. Ioo environ de l'azote ingéré en plus. Au bout de $24 \mathrm{~h}$, une quantité relativement importante (30 à $80 \mathrm{p}$. IOo) reste apparemment retenue par l'organisme. Les croissances effectuées par les porcs entraînent à penser que cet azote pourrait être partiellement utilisé. Une telle hypothèse est en cours de vérification.

\section{Azote urinaire non uréique et non ammoniacal.}

Par contre, il apparaît, et ceci est surtout net avec le régime HN à $8 \mathrm{p}$. Ioo, que les émissions urinaires d'azote non uréique et non ammoniacale ( $N$ non ur-amm) des porcs N.P.R. sont maxima, lorsque le porc $5^{B}$ manifeste l'économie maxima de ses dépenses, et que ces émissions reviennent après $r 8 \mathrm{~h}$. au niveau observé après le repas $\mathrm{BN}$.

La simultanéité de l'augmentation des émissions urinaires d'azote non ur-amm et de la diminution des dépenses énergétiques postprandiales induites par un repas $\mathrm{HN}$ et leur retour vers la même période aux valeurs des repas $\mathrm{BN}$, fait supposer qu'un ou des métabolites azotés présents dans cette fraction seraient à l'origine de l'économie de calories. Ces métabolites pourraient-ils être des acides aminés ? Leur présence en très petite quantité dans l'urine a été démontrée depuis longtemps en repas normal (LEVENE et VAN SLYKE, I9I2) et leur augmentation serait expliquée par celle de l'aminoacidémie sanguine. En effet PION etal. (I963) montrent que chez de jeunes porcelets, l'aminoacidémie porte subit des variations importantes dans les $2 \mathrm{~h}$ qui suivent le repas et que toute augmentation de la richesse en azote des régimes l'accroît fortement. Une telle hypothèse demanderait une vérification précise. En effet, dans l'expérience actuelle, l'évaluation de cette fraction azotée est faite par différence, elle est donc passible d'erreurs assez importantes et les mesures d'énergie et les excrétions d'azote ont été effectuées sur des animaux différents. Mais de nombreux autres produits se trouvent aussi dans cette fraction et pourraient jouer un rôle.

\section{Rapidité des réactions métaboliques animales.}

La diminution des dépenses d'énergie consécutive au repas $H N$ par rapport au repas $B N$ est déjà importante une heure après le repas : I2 à 28 p. Ioo. La différence maximale est atteinte $I$ à $2 h$ après le repas.

Cẹtte rapidité semble normale. Chez des porcs de $95 \mathrm{~kg}$ environ, qui ingèrent des régimes de composition voisine des nôtres, RÉRAT et LOUGNON (I963) constatent que la disparition de l'azote alimentaire dans l'estomac atteint 33 à 50 p. Ioo respectivement $\mathrm{I}$ à $2 \mathrm{~h}$ après le repas. Cette rapidité de transit conditionne l'arrivée des protéines dans le duodénum et ultérieurement le passage des formes hydrolysées dans la circulation sanguine. Comme nous venons de le signaler PIon et al. (1963) montrent la rapidité des variations de l'aminoacidémie porte chez de jeunes porcelets.

De même, chez le Rat, DAwson et al (I962, I et 2) travaillant avec des chlorelles marquées au ${ }^{14} \mathrm{C}$ constatent que $\mathrm{I} h$ après le repas, $35 \mathrm{p}$. Ioo des protéines alimentaires ont disparu du tractus digestif et mesurent, simultanément, des activités spécifiques dans les fèces, l'urine, la muqueuse intestinale, le foie et même la carcasse éviscérée; le marquage maximum des aminoacides du sang systémique a lieu I à $2 \mathrm{~h}$ après le repas soit un peu plus tard que celui du sang porte. Donc les phéno- 
mènes de digestion, en particulier de vidange stomacale et de catabolisme azoté, même s'ils sont différents pour les repas $\mathrm{HN}$ et $\mathrm{BN}$, sont toujours suffisamment rapides pour que des produits de ce catabolisme puissent être à l'origine des variations de dépenses d'énergie.

\section{2. - Abaissement des quotients respiratoires}

Les moindres dépenses postprandiales d'énergie dues aux repas HN par rapport aux repas $B N$ s'accompagnent d'un abaissement des Q.R. et ceci quelles que soient les conditions alimentaires et le poids des sujets. Comment peut-on interpréter cette chute.

\section{TABLEAU 3}

Variations des quotiens respiratoires au cours de la journée selon le repas

\begin{tabular}{|c|c|c|c|c|c|c|c|c|c|c|}
\hline \multirow{2}{*}{ Porcs } & \multirow{2}{*}{ Repas } & \multicolumn{8}{|c|}{ Temps en minutes après le repas } & \multirow{2}{*}{$\begin{array}{l}\% \text { moyen } \\
\text { de chute }\end{array}$} \\
\hline & & 60 & 90 & 120 & 180 & 240 & 300 & 360 & 420 & \\
\hline \multirow[t]{2}{*}{$1_{A}$} & $\mathrm{BN}$ & 1,051 & 1,036 & 1,086 & 1,091 & 1,070 & 1,061 & 1,051 & 1,025 & \\
\hline & $\mathrm{HN}$ & 0,896 & 0,816 & $0,86^{\prime} t$ & 0,905 & 0,897 & 0,875 & 0,836 & 0,839 & -18 \\
\hline \multirow[t]{2}{*}{$1_{B}$} & $\mathrm{BN}$ & 1,121 & 1,099 & 1,051 & 1,115 & 1,075 & 1,014 & $0,96 x$ & 0,986 & \\
\hline & $\mathrm{HN}$ & 0,871 & $0,75^{\prime} \mathrm{t}$ & 0,771 & 0,944 & 1,005 & 1,023 & 1,041 & 1,000 & -11 \\
\hline \multirow[t]{2}{*}{$\ddot{z}_{\mathrm{A}}$} & $\mathrm{BN}$ & 0,8688 & 0,926 & $0,93^{\prime} \mathbf{t}$ & 0,890 & 0,935 & 0,972 & 1,001 & 0,967 & \\
\hline & $\mathrm{HN}$ & 0,882 & $0,80^{\prime}$ & 0,816 & 0,905 & 0,929 & 0,953 & 0,971 & 0,897 & - ${ }_{t}^{\prime}$ \\
\hline \multirow[t]{2}{*}{$-2_{B}$} & $\mathrm{BN}$ & - & 0,863 & 0,888 & 0,998 & 0,957 & 0,900 & 0,835 & 0,851 & \\
\hline & $\mathrm{HN}$ & 0,785 & 0,766 & 0,796 & 0,929 & 0,903 & $0,86^{\prime} t$ & 0,920 & 0,831 & - $\quad$ f \\
\hline \multirow[t]{2}{*}{$3_{\mathrm{A}}$} & $\mathrm{BN}$ & $1,06 / t$ & 1,051 & 1,083 & 1,046 & 1,049 & $1,0 / 9$ & 1,027 & 0,977 & \\
\hline & $\mathrm{HN}$ & 0,881 & 0,889 & 0,883 & 0,857 & 0,864 & 0,864 & 0,881 & 0,897 & -16 \\
\hline \multirow[t]{2}{*}{$3_{\mathbf{B}}$} & $\mathrm{BN}$ & 1,213 & 1,109 & $1,12^{\prime}$ & 1,156 & $1, \mathbf{1 6 0}$ & 1,160 & 1,173 & 1,237 & \\
\hline & $\mathrm{HN}$ & 0,867 & 0,902 & 0,919 & 0,938 & 0,955 & $0, \$ 70$ & 0,967 & 0,936 & $-\because 0$ \\
\hline \multirow[t]{2}{*}{$i_{A}$} & $\mathrm{BN}$ & 1,011 & $1,06^{\prime}$ & 1,079 & 0,932 & 1,081 & 1,095 & 1,110 & $1,1: 5$ & \\
\hline & $\mathrm{HN}$ & 0,883 & 0,905 & 0,910 & 0,842 & 0,855 & 0,867 & 0,910 & 0,958 & -16 \\
\hline \multirow[t]{2}{*}{${ }^{\prime} \mathrm{B}$} & $\mathrm{BN}$ & 1,126 & 1,080 & 1,088 & 1,051 & 1,019 & 0,982 & 0,986 & $1,03^{\prime}$ & \\
\hline & $\mathrm{HN}$ & 0,935 & 0,932 & 0,896 & 0,851 & 0,892 & $0,9 y^{\prime} \mathbf{0}$ & 0,981 & 0,952 & -12. \\
\hline 5 & BN & 1,185 & 1,278 & 1,226 & 1,256 & 1,276 & $1, \geq 90$ & 1,270 & 1,220 & $\cdots$ \\
\hline $\mathrm{A}$ & $\mathrm{HN}$ & 1,230 & 1,230 & 1,180 & 1,166 & 1,210 & 1,250 & 1,253 & 1,216 & -3 \\
\hline B & $\mathrm{HN}$ & 1,006 & 1,022 & 1,033 & 0,935 & 0,946 & 0,967 & 0,999 & 0,953 & -21 \\
\hline \multirow[t]{2}{*}{6} & $\mathrm{BN}$ & 1,027 & 1,062 & 1,085 & 1,118 & 1,095 & 1,070 & 1,065 & 1,086 & \\
\hline & $\mathrm{HN}$ & 0,882 & 0,918 & 0,906 & 0,882 & 0,856 & $0,83^{\prime}$ & 0,833 & 0,798 & -19 \\
\hline
\end{tabular}

L'importance de la lipogenèse chez le Porc est particulièrement forte puisque déjà à $30 \mathrm{~kg}$, le coût énergétique de sa lipogénèse est égal à celui de sa protéinogenèse (OsLAGE et al., ThORBECK). Les réactions de décarboxylation oxydative qui sont à l'origine de la synthèse des graisses à partir des glucides, s'opèrent avec des Q.R. toujours très élevés, aussi les Q.R. moyens observés par les auteurs précités sont sur des bilans de $24 \mathrm{~h}$, même à ce poids, égaux ou très légèrement supérieurs à $\mathrm{I}$.

Mais au cours de la journée, en dehors des variations provoquées par les modifi- 
cations du pool carboné de l'organisme, les Q.R. ne sont pas constants. Inférieurs ou égaux à I le matin selon l'âge des animaux, ils sont beaucoup plus élevés durant la phase postprandiale (données personnelles non publiées). Ils peuvent atteindre jusqu'à $I, 3$ à $I, 5$, valeurs que l'on retrouve ici pour le Porc $5(60 \mathrm{~kg})$ consécutivement aux repas $\mathrm{BN}$ (données brutes), et qui ont déjà été signalées en 1925 par WIERzUchowski et LiNG.

Plusieurs hypothèses qui ne s'éliminent pas mutuellement peuvent être formulées pour expliquer l'abaissement des $Q . R$. pendant plusieurs heures après l'ingestion des repas $\mathrm{HN}$.

L'aminoacidémie intense qui a obligatoirement lieu après un repas $\mathrm{HN}$ pourrait être à l'origine de deux séries d'action. Il semble que certains acides aminés (leucine, arginine, tryptophane) aient une action hypoglycémiante au niveau sanguin ce qui expliquerait le ralentissement et même l'arrêt de la lipogenèse à ce moment. Des perfusions veineuses d'acides aminés provoquent en effet une chute de la glycémie et d'importantes élévations de l'insulinémie, (RAU'TUREAU, I968). D'autre part, cette même aminoacidémie provoque obligatoirement un catabolisme beaucoup plus élevé que celui dû aux repas $B N$, qui se traduit par une augmentation beaucoup plus forte de l'excrétion d'azote total et uréique (fig. 2 et 3). Or, selon HENRY (I934), 1'uréogenèse à partir de l'alanine s'accompagne chez les mammifères d'un Q.R. égal à o,83. Cette dernière hypothèse trouve une confirmation dans le maintien des différences des Q.R. jusqu'à la fin des mesures $(420 \mathrm{mn})$ mais le maintien à un niveau élevé de l'excrétion d'urée au cours de la nuit suivante entrainerait que les Q.R. restent relativement bas durant toute cette période, ce qui n'a pas été vérifié puisque nous avons limité la durée de nos mesures à 7 heures.

Enfin les repas HN sont évidemment moins riches en glucides que les repas BN et comportent peu de formes rapidement utilisables. La glycémie postprandiale doit logiquement être assez faible et l'organisme animal pourrait être conduit, pour faire face à ses besoins immédiats en glucides solubles, à synthétiser des glucides à partir de ses lipides corporels, comme l'ont montré Weinman et al. (1957). Or, cette synthèse s'effectue avec $Q . R$. inférieur à $I$. Une phase de lipolyse pourrait-elle avoir lieu après l'ingestion du repas HN? En comparant des rats nourris normalement à des rats soumis à des phases de jeûnes hydriques intermittents, PATRASEK et al. (I964) constatent dans les expériences in vitro que le foie des seconds serait plus apte à oxyder les acides gras que celui des premiers; et sur des rats soumis alternativement à des périodes d'alimentation normale et de restriction (40 p. IOO) Morin-Jomain (I962), observe un abaissement des dépenses d'énergie et des Q.R. plus faibles en périodes de restriction $(0,85)$ tandis qu'en période de réhabilitation, ceux-ci sont proches de $\mathrm{I}$. Cependant après 5 alternances, les sujets restreints sont légèrement plus gras que les normaux. Il en est de même de rats soumis à des jeûnes protéiques intermittents (Morin-Jomain, I963). Mais l'alternance des régimes sur de longues périodes n'est pas comparable à l'apport massif que nous avons effectué 2 fois par semaine.

$$
\text { 3. - Effet de choc }
$$

On connaît l'influence généralement positive de la richesse en protéines du régime auquel est soumis l'animal sur ses dépenses énergétiques du métabolisme basal. W'IsHAR'T (I928), KROGH et LINDHARD (I920) l'ont observée chez 1'Homme, HorsT et al. 
(I934) chez le Rat, tandis que le Chien n'y semble pas sensible ANDERSON et Lusk, (I9I 7) ; CharletT-Lery (I96I).

Chez le Porc, les données que nous avons relevées (I966) concernent le métabolisme d'entretien (le matin, ${ }_{5} \mathrm{~h}$ après le précédent repas). Celui-ci est lié à la richesse en azote du régime par un coefficient de corrélation de $+0,74 \pm 0,08$.

Mais toutes ces observations ont été faites après une longue adaptation des animaux à leur régime.

Ici même, la richesse azotée du régime $\mathrm{BN}$ que le porc consomme régulièrement, provoque un niveau de dépenses supérieures. Ceci ressort nettement de l'ensemble des figures I et des données du tableau 2. Par exemple, les dépenses horaires du sujet 2 sont en moyenne légèrement supérieures à $3 \mathrm{kcal} / \mathrm{kg}$ vif lorsqu'il ingère le régime $\mathrm{BN}$ à $0,80 \mathrm{p}$. Ioo $\mathrm{N}$ et avoisinent $4 \mathrm{kcal}$ avec celui à $3,80 \mathrm{p}$. Ioo.

Ce rôle de la richesse en protéines des régimes chez le Porc après adaptation s'oppose donc très nettement à la diminution des dépenses après ingestion brutale d'un repas $\mathrm{HN}$, le porc subirait peut-être un choc protéique.

Ce choc protéique semble d'autant plus aisé à admettre que son existence se manifeste quel que soit le rôle que $\mathrm{N} i+\mathrm{d} u$ repas $\mathrm{HN}$ a été susceptible de jouer : combler une carence ou être excédentaire. En effet, les animaux 2, 3 et 4 reçoivent successivement des régimes $\mathrm{BN}$ qui les mettent dans des conditions de carence protéique $\left(0,8 \mathrm{~N}\right.$ p. Ioo M.S.). et dans des conditions d'équilibre $\left(2,55^{-2,67} \mathrm{~N}\right.$ p. Ioo M.S.). Or, le nombre de calories économisées par $\mathrm{g} \mathrm{Ni}+$ reste pratiquement inchangé ; il s'élève respectivement à 2,39 et 2,40 pour le porc 2 , à 2,08 et 2,65 pour le 3 et à 2,69 et $2,6 \mathrm{I}$ pour le 4 . Ce choc coïnciderait avec l'apparition d'un métabolite dont on décèle la présence dans la fraction non ur-amm de l'azote urinaire et pourrait s'effectuer par voie hormonale.

Malgré la répétition des mesures sur un même animal, aucune adaptation ne semble intervenir. En effet, chaque porc ingère 8 à Io repas HN dont les premiers servent à contrôler l'appétibilité des rations et les derniers aux mesures. Le nombre des repas HN n'excédant pas 2 par semaines, la durée d'un essai sur un animal est de 4 à 6 semaines, durée suffisamment longue pour que se manifeste une adaptation si elle devait avoir lieu.

Les hypothèses actuellement admises qui s'appliquent parfaitement aux observations que nous avons faites antérieurement chez le Chien ne semblent pas convenir pour expliquer l'ensemble des faits observés chez le Porc en croissance.

Il semble donc judicieux de rechercher un supplément d'information par l'étude d'un certain nombre de phénomènes concomitants. Parmi ceux-ci, nous comptons aborder l'étude du niveau de vigilance (sommeil) et de certains paramètres physiologiques chez les animaux soumis à l'alternance des repas BN et $H N$ ce qui devrait nous aider dans la recherche du ou des métabolites susceptibles d'être à l'origine de cette diminution de dépenses. Mais leur action pourrait s'expliquer soit par dépassement d'un seuil - les quantités $\mathrm{N} i+\mathrm{P}^{\mathbf{0 7 0}}$ ingérées par le porc en croissance étant très élevées - soit être caractéristique d'une espèce, ce qui nous obligerait à faire quelques observations sur des porcs adultes ingérant des rations d'entretien.

En outre, la mesure de bilans azotés sur des animaux soumis à cette alternance de régimes permettra de préciser le sort de l'azote ingéré dans les repas HN. 


\section{SUMMARY}

\section{VARIATIONS IN HEAT INCREMENT CAUSED BY INGESTION OF MEAIS VERY RICH IN PROTEIN BY GROWING PIGS}

After feeding, growing pigs of 30 to $60 \mathrm{~kg}$ liveweight show the normal extra-heat which, we have measured in numerous short-term experiments by indirect calorimetry. Ingestion of a very high protein feed $(\mathrm{HN})$ compared to a normal equienegetic one (BN) induces, in pigs a post-feeding energetic losses.

This reduction in expenditure is contrary to all the previous observations, in particular to those on dogs. It was maximum after one or two hours ( -12 to 28 per cent), it lasted 6 to 7 hours and was accompanied by a fall in respiratory quotient (-I 2 per cent).

This saving of energy was proportional to the amount of extra protein ingested $(2.34 \pm$ o.ro kcal per $\mathrm{g}$ extra $\mathrm{N}$ ingested, $r=0.98)$, or retained in the body during the day $(3.35 \mathrm{kcal}$ per $\mathrm{g}$ extra $\mathrm{N}$ retained). It did not seem to be correlated to total $\mathrm{N}$ in urine nor to the urea or ammonia fractions eliminated during the day ( $9 \mathrm{~h}$ to $\mathrm{I} 8 \mathrm{~h}$ ). This "protein stress", which occurred whatever the role played by the excess protein (HN-BN) - whether avoiding to deficiency or giving rise to an excess - could be related to the appearance in urine of a metabolite present in some fraction other than urea or ammonia, an appearance which would coincide with the reduction of energy losses.

The high amino acid value in blood which necessarily follow the ingestion of the feed verg rich in $\mathrm{N}$ could explain at least in part the lowering of respiratory quotient, of wich values below I indicate that lipogenesis has stopped during this time.

\section{RÉFÉRENCES BIBLIOGRAPHIQUES}

Anderson R. S., Lusk G., x917. The interrelation between diet and body condition and the energy production during mechanical work. J. Biol. Chem., 32, $42 \mathrm{I}$.

Brody S., I945. Bioenergetics and growth, 59-74, Reinhold Pub. Corporation, New York.

Brouwer E., I958. On simple formulae for calculating the heat expenditure and the quantities of carbohydrates and fat metabolized in ruminant fromdata on gazeous exchange and urine N. Proceed. 1st Symp. Energy Metabolism. 182-192.

Charlet-LeRY G., I958. a) Précisions analytiques obtenues avec certains appareils de dosages physiques (Beckman $\mathrm{O}_{2}-$ Analyseur infra-rouge $\mathrm{CO}_{2}$ ). b) Méthode de mesure de courte durée des échange gazeux.

Proceed 1st Symp.Energy Metabolism 124-126, 194-202.

Charlet-Lfry G., I96r. Relation entre action dynamique spécifique et niveau de consommation de matière sèche et d'azote. Proc. 2 nd Symp. Energy Metabolism. I77-186.

Charlet-Lery G., r966. Étude critique de la notion d'action dynamique spécifique chez le porc en croissance. IX $X^{\mathbf{e}}$ congrès international de Zootech. Programme scientifique et résumés. $\mathbf{3} 6$.

Charlet-lery G., r97o. Influence de la dissociation partielle des ingestions d'energy et de protéines sur la croissance et les bilans d'azote chez le pore en croissance. Ann. Biol. anim. Bioch. Biophys. sous presss.

Dawson R., Holdsworth E. S., Ig62. An investigation into protein digestion with 14-C labelled protein. I. The general pattern of ${ }^{14} \mathrm{C}$. incorporation in body tissues and fluids of the rat up to $3 \mathrm{~h}$ after feeding Brit. J. Nut., 16, $13-26$.

Dawson R., Porter J. W. G., Ig62, 2, - The transport of ${ }^{14} \mathrm{C}$ labelled nitrogenous compounds in the rat and cat. Brit. Brit. J. Nutr., 16, 27-39.

FABRY P., r967. Metabolic consequences of the pattern of food intake Handbook of Physiology Ali. mentary Canal, 1, 3I-49. American Physiological Society, Washington.

Forbes E. B., Swift R. W., I944. Associative dynamic effects of protein, carbohydrate and fat. $J$. Nutr., 27, 453-468.

Henry K. M., Magee H. E., Reide, I934. Some effects of fashing on the composition of the blood and respiratory exchange in fowls. J. Biol., 11, 58-72.

Horst K., Mendel L. B.., Benedict F. G., I934. The influence of previous diet, growth and age upon the basal metabolism of the rat. J. Nutr. 8, 139-162.

KLeiber M., I96r. The fire of life, 266-27r. John Wiley and Son, New York, Londres. 
KROGH A., LindhaRd J., 1920. The relative value of fat and carbohydrate as sources of muscalar energy. With appendices on the correlation between standard metabolism and the respiratory quotient during rest and work. Bioch. J., 14, 290.

Levene P. A., VAN Slyke D. D., I9I2. Gasometric determination of free and conjugated amino acids in the urine. J. Biol. Chem., 12, 301.

Mrtchell H. H., I955. The specific dynamic action of food. Scientia (Milan), 90, I93-I99.

Mitchell H. H, 1964. Comparative nutrition of man and domestic animals vol. II, 471-565. Academic Press, New York, Londres.

Oslage H. J., Fliegel H., Farries F. E., Richter K., I966. Stickstoff, Fett und Energieansatz bei wachsenden Mastschweinen. Zeitsch. Tierphys. Tiernäh Futtermittel, 21, 50-65.

Petrasek R., Fabry P., Poledne R., i 964 . Effect of feeding pattern on fatty acid oxidation by rat liver slices. Experientia, 20, 434-435.

Pron R., Fauconneau G., Rérat A., ig63. Étude cinétique de la composition en acides aminés du sang porte chez le Porc. Ann. Biol. anim. Bioch. Biophys., 3, no hors-série, 3I-37.

Ravtureau J., 1968. Hypoglycémie et acides aminés. Cahiers Nutr. Diet., 3, 59-64.

Rérat A., Lougnon J., I963. Études sur le transit digestif chez le Porc. Ann. Biol. anim. Bioch. Biophys., 3, no hors-série, 2 I-29.

Schaeffer G., Lebreton E., x938. L'action dynamique spécifique des protides, vol. I, Hermann, Paris. SchoenheINer R., 1942. The dynamic state of body constituants. Harward Univ. Press.

Thorbek Grete., 1967. Studies on energy metabolism of growing pigs. 4th symposium on Energy metabolism. $28 \mathrm{I}-\mathrm{I} 89$.

Weinman E. O., Strisower E. H., Chaikoff I. L., I957. Conversion of fatty acids to carbohydrate: application of isotopes to this problem and role of the Krebs cycle as a synthetic pathway. Physiol. Revs, 37, 252-272.

Wierzuchowski M., LiNG S. M., 1925. Animal calorimetry. 29th paper on fat production in a young hog. J. Biol. Chem., 64, 697-707.

Wishart G. M., I928. The influence of the protein intake of the basal metabolism. J. Physiol., 65, $243-254$.

Yeo M. L., Chamberlain A. G., I966. Delayed protein supplementation of barley diets for weanling pigs. Proc. Nut. Soc., 25, 2 XII. 